\title{
The Use of Topical Cream Anesthetics in Office Procedures of the External Genitalia
}

\author{
Kostis Gyftopoulos \\ Urologic Surgeon, Olympion Hospital and Department of Anatomy, University of Patras
}

Greece

\section{Introduction}

The use of anesthesia during nineteenth century was extremely limited due to the hazards of the available anesthetics and the inability to successfully monitor the patient. This resulted in the majority of surgical procedures being performed without any anesthesia (Gordetsky et al, 2011). A revolutionary step in the history of anesthetics began in 1884, when Carl Koller, a scientist from Vienna, experimented with topical application of cocaine in the eye, achieving adequate surgical analgesia, without the potentially lethal side effects of inhalation anesthesia (Neilson, n.d.). The use of this natural alkaloid provided results superior to the contemporary general anesthesia and surgeons quickly appreciated the advantages of local anesthetics. Especially urologists embraced rapidly the promising local anesthetic and began experimenting with cocaine as a local agent for urological procedures, mainly by topical and intraurethral application (Gordetsky et al, 2011). It took a while for the initial enthusiasm to settle down as reports for side effects and addiction were bringing debate in the medical community. The need for less toxic drugs would lead to synthetic local drugs such as procaine; the discovery of the stable amide lignocaine in 1948 by Lofgren was an important milestone in the development of safe and effective local anesthetics.

A plethora of urological or gynecological surgical procedures are performed nowadays under local anesthesia at an office setting. In the male these minor procedures usually deal with the prepuce or the urethral meatus (separation of preputial adhesions, short frenulum plasty, meatotomy, modified or complete circumcision). In the female, these procedures deal with lesions of the vulvar skin or the cervix (vulvar or vaginal biopsy, laser treatment of vulvar and cervical lesions, hysteroscopy). Removal of genital warts (condylomata accuminata) is also a common procedure under local anesthesia in both genders. The need for some form of anesthesia is important for both the patient and the surgeon. For the patient it is a human right not to suffer pain or distress associated with a surgical procedure. On the other hand, withholding analgesia may affect the quality of the surgical outcome, as the patient's anxiety, reaction to pain and improper movement may complicate the surgeon's ability to perform a delicate procedure. This is especially true in the above mentioned procedures due to the unique sensitivity of the external genitalia.

The diverse innervation of the genital skin, especially the penile and clitoral prepuce, may account for the increased discomfort during procedures of the external genitalia in both sexes. The penile and vulvar skin and mucosal surfaces are highly sensitive to pain (Zilbert 
2002, Cold \&Taylor, 1999). The somatosensory innervation of the genitalia is complex and still under debate. The male prepuce receives branches from the dorsal nerve of the penis and branches of the perineal nerve. Some sensory pathways of the genital tract may even by-pass the spinal cord through the vagus nerve. Moreover, the genital skin and especially the prepuce is rich in encapsulated somatosensory receptors, which include Meissner's corpuscles, Vater-Pacinian corpuscles and Merkel cells. These extremely sensitive receptors are probably part of the erogenous function of the genital skin (Cold \& Taylor, 1999). Additionally, free nerve endings account for the protopathic sensitivity i.e. crude, poorly localized feelings which however include pain. This complex pattern of innervation not only explains the high sensitivity of the genital region but also emphasizes the need for adequate anesthesia for the performance of surgical procedures.

The most common way of achieving anesthesia in the genital area is infiltration of a local agent. This is a logical option for the majority of the afore-mentioned procedures, as they are relatively superficial. Local infiltration obviates the need for sedation and the risks of other forms of anesthesia; however it can still cause discomfort to the patient. Injections of local anesthetics are painful by themselves, may worsen needle anxiety and increase pain perception (Kaweski, 2008). Moreover they may cause bleeding and distort the surgical area by development of oedema or haematoma. There is also the risk of inadvertent intravascular injection, especially when larger volume of anesthetic solution is used. Topical anesthetic creams may in some cases replace injected local anesthetics. They are much more "patient-friendly", as they eliminate needle fear and pain in the beginning of the procedure. It has been shown that by using a topical anesthetic cream the patient's anxiety and distress of the procedure itself are alleviated. This is also valuable in children, as the pain factor and the increased pain perception are overcome with the application of a topical cream (Kaweski, 2008).

We hereby describe our experience on the efficacy and safety of a topical anesthetic cream (EMLA, Eutectic Mixture of Local Anesthetics, 2.5\% lidocaine and 2.5\% prilocaine) as topical anesthetic in several office surgical procedures of the external genitalia in both males and females.

\section{Patients and methods}

During a period of 8 years (2003-2010) a total of 321 patients were subjected to minor surgery of the external genitalia at an office setting under local anesthesia. The range of procedures included: Separation of preputial adhesions in boys, complete circumcision for phimosis, short frenulum plasty, meatotomy, fulguration of genital warts (penile, vulvar), fulguration of urethral (meatal) warts and excision of urethral prolapse in women. The distribution of cases is described in Table 1.

All patients were offered to choose between infiltration with a local anaesthetic and application of a topical cream as a form of local analgesia. After detailed information was given, all patients opted for the topical cream application. Informed consent was obtained by those patients whose genitals were photographed for archival and scientific purposes.

In all cases the topical cream used was EMLA 5 gr (Eutectic Mixture of Local Anesthetics, $2.5 \%$ lidocaine and $2.5 \%$ prilocaine), AstraZeneca, Inc. The amount of cream used varied among individuals and procedures (see below). For the fulguration of genital warts, 
frenulum plasty and haemostasis during circumcision we used the Birtcher Hyfercator PlusTM (Birtcher Medical Systems Inc, Utica, NY), a high frequency device used for monoterminal fulguration at low power settings (7-10 Watts).

\begin{tabular}{|c|c|c|c|c|}
\hline PROCEDURE & No & MALE & FEMALE & AGE (mean \pm SD) \\
\hline Preputial adhesions & 17 & 17 & & 52 months \pm 18.7 \\
\hline Circumcision & 68 & 68 & & $47.8 \mathrm{yrs} \pm 24.7$ \\
\hline Short frenulum & 152 & 152 & & $24.9 \mathrm{yrs} \pm 4.7$ \\
\hline Meatotomy & 12 & 12 & & $56.1 \mathrm{yrs} \pm 28.4$ \\
\hline Genital warts & 48 & 42 & 6 & $23.9 \mathrm{yrs} \pm 3.3$ \\
\hline Urethral warts & 17 & 17 & & $24.5 \mathrm{yrs} \pm 5.8$ \\
\hline Urethral prolapse & 7 & & 7 & $74.8 \mathrm{yrs} \pm 6.5$ \\
\hline
\end{tabular}

Table 1. Distribution of patients according to surgical procedure.

\subsection{Application method and surgical procedure}

\subsubsection{Separation of preputial adhesions}

Separation of preputial adhesions was performed in 17 boys (mean age \pm SD: 52 months \pm 18.7). The procedure was reserved only for boys with partial preputial adhesion and history of previous inflammation (balanoposthitis) or previous urinary tract infection. Patients with verbal ability were thoroughly informed of the procedure and reassured that they would feel no pain. A small amount of EMLA (0.5-1gr) was smeared inside the preputial sac using a cotton tip. Children were allowed to relax for 20 minutes in the waiting room for the anesthetic to act. The procedure was accomplished by gentle traction of the prepuce and concomitant separation of the adhesions using a fine haemostatic clamp (mosquito clamp). Special care was taken not to force the separation of the fused mucosa, in order to avoid bleeding and glanular excoriation. After completion of the procedure, a small quantity of povidone iodine cream was applied and the prepuce was pulled back to cover the glans. The young boys were kept for one hour in the waiting room for monitoring and dismissed after thorough consultation of the parents on possible adverse effects during the following hours. Parents were advised to start local hygiene with a mild soap on the next day, gently retracting the foreskin. No further antiseptics or antibiotics were prescribed.

\subsubsection{Short frenulum plasty}

Short frenulum plasty was performed in 152 young males (mean age \pm SD: 24.9 yrs \pm 4.7 ). The method used for short frenulum correction was the "pull and burn method" described previously (Gyftopoulos, 2009). Briefly, a small amount of EMLA cream (max 2 gr) was applied at the frenulum area and an occlusive dressing (Tegaderm ${ }^{\circledR}, 3 \mathrm{M}$ Inc.) was used to prevent the cream from leaking. After 20 minutes the adhesive dressing was gently removed, excess cream was cleaned, the glans was smeared with povidone iodine and the procedure started if the patient reported no pain at all at pinprick. By retracting the glans upwards, the point of maximum tension on the frenulum was noted (Fig. 1). A small horizontal cut was made using the Hyfercator and the superficial layer of the frenulum was gradually separated by applying gentle and stable traction to the glans and the shaft of the 
penis. Minor bleeders were sealed by the Hyfercator at low power, taking care not to severe the frenular artery that was now lying at the frenular bed. When full relaxation of the frenular chordee was achieved a small quantity of povidone iodine cream was applied on site and the prepuce was pulled back to cover the glans. Patients were instructed to start personal hygiene on the following day, with emphasis on keeping the trauma as dry as possible (e.g. by retracting the foreskin when voiding). No antibiotics were given and sexual abstinence was suggested for at least 15 days.

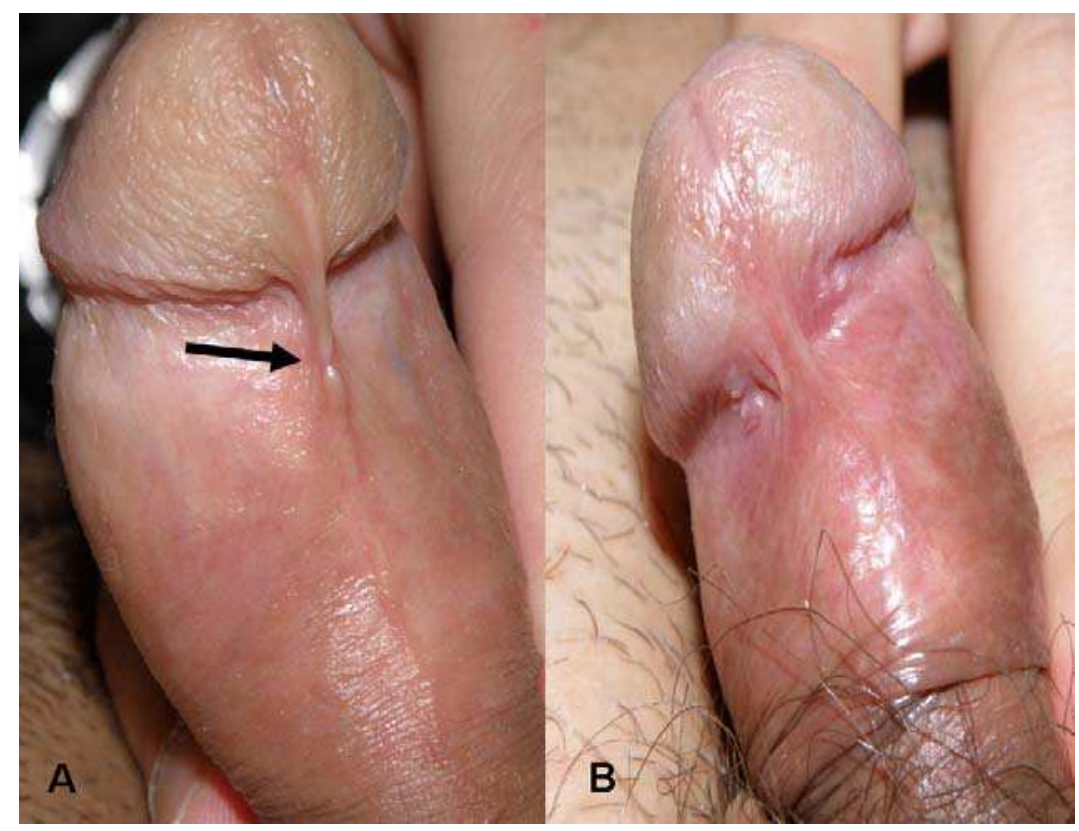

Fig. 1. Short frenulum cases. Note previous scar from trauma in case A (arrow).

\subsubsection{Meatotomy}

A total of 12 patients (mean age \pm SD: 56.1 yrs \pm 28.4 ) were subjected to meatotomy due to meatal stenosis. In four cases the patient had Balanitis Xerotica Obliterans (BXO) affecting the glans and the meatus. A small quantity of EMLA cream ( 1 gr) was applied using a small $1 \mathrm{ml}$ syringe. An occlusive dressing (Tegaderm ${ }^{\circledR}, 3 \mathrm{M}$ Inc.) was used to prevent the cream from leaking. After 20 minutes the adhesive dressing was gently removed, the glans was squeezed to remove excess cream and a small haemostatic clip was used to spread the urethral meatus. An incision was made to the ventral surface of the meatus using the lancet of the Birtcher Hyfercator. The incision was extended ventrally ( 6 o'clock position) until a sufficient opening of the meatus was achieved. This was calibrated using the tip of a $18 \mathrm{Fr}$ Tiemann catheter. In 4 patients the urethral mucosa was sutured at the meatal lips at the 3 and 9 o'clock position using fine absorbable sutures (5-0 polyglycolic acid, Safil Quick ${ }^{\mathrm{TM}}, \mathrm{B}$. Braun Melsungen AG). No postoperative analgesia was given. The patients were advised to avoid irritative food and liquids (e.g. pepperoni, alcohol consumption) for the following days. Local hygiene (mild soap and water) was allowed from the following day. 


\subsubsection{Fulguration of urethral warts}

A total of 17 male patients (mean age \pm SD: 24.5 yrs \pm 5.8 ) were treated for warts (HPV lesions, condylomata acuminata) at the external urethral meatus. The lesions were limited to the meatus or fossa navicularis. A small quantity of EMLA cream $(\sim 1$ gr $)$ was applied using a small $1 \mathrm{ml}$ syringe (Fig. 2). An occlusive dressing (Tegaderm ${ }^{\circledR}, 3 \mathrm{M}$ Inc.) was again used to prevent the cream from leaking. 20 minutes later the adhesive dressing was removed, the glans was squeezed to remove excess cream and a small haemostatic clip was used to spread the urethral meatus. The warts were fulgurated using the Birtcher Hyfercator at low power settings (approximately 5 Watts). In 7 cases $(41 \%)$ there were multiple HPV lesions (up to three) inside the urethral meatus. All of these lesions were treated in one session.Suspicious reddish areas of the urethral mucosa, when present, were also fulgurated; however care was taken to limit the fulgurated area to approximately $40 \%$ of the mucosal surface in order to avoid possible meatal stenosis. In 5 cases a modified meatotomy was necessary in order to have access to the lesions. No postoperative analgesia was given. The patients were advised to consume at least 2 liters of clear water daily, to avoid irritative food and liquids (e.g. pepperoni, alcohol consumption, acidic refreshments) and to void frequently for the following week. Local hygiene (mild soap and water) was allowed from the following day but sexual abstinence was advised for at least 10 days; condom use was suggested after that period. The patients were also advised to inspect the meatal opening for possible recurrence during the healing period.

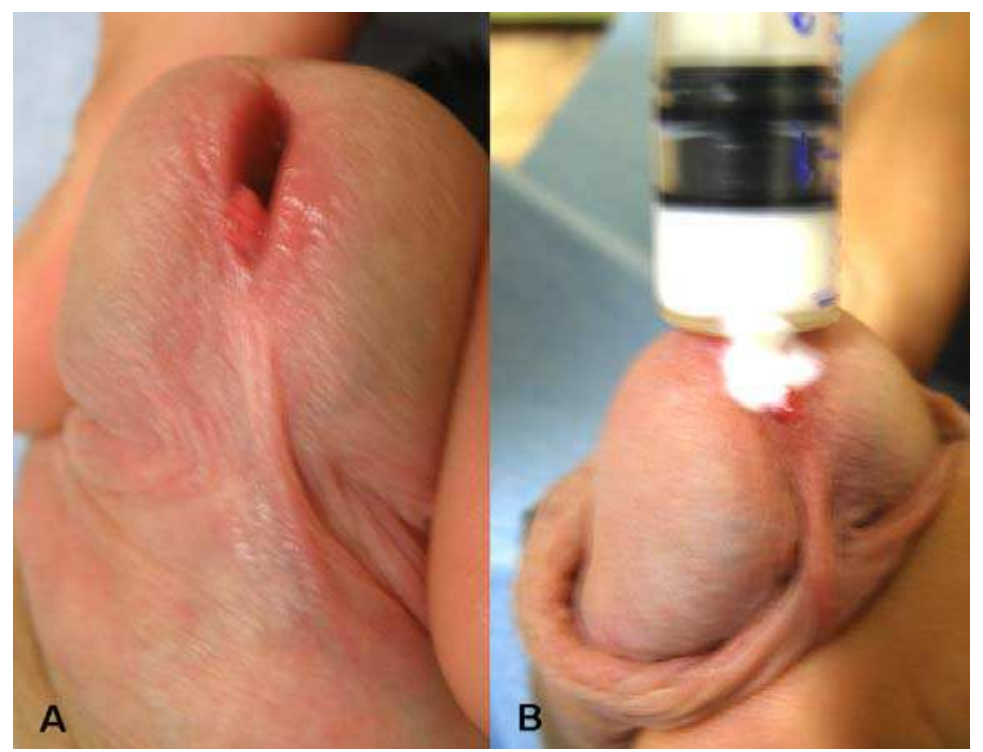

Fig. 2. HPV lesion protruding from the urethral meatus (A). Topical cream applied with a syringe (B).

\subsubsection{Urethral prolapse correction}

Seven elderly female patients (mean age \pm SD: 74.8 yrs \pm 6.5 ), presenting with urethral prolapse were treated under topical anesthesia. After preparation of the genitalia with povidone iodine, 
the EMLA cream (approximately 2 gr) was applied inside the urethral mucosa using a small 5 $\mathrm{ml}$ syringe. The external meatus and parameatal tissue was also smeared with a small quantity of cream. No dressing was used but the patient was left in lithotomy position for 20 minutes. Subsequently the excess cream was removed, the prolapsed mucosa was grasped with an Allis forceps and the Birtcher Hyfercator was used to cut the prolapsed mucosa. Any bleeding vessels were sealed at the same time. After excision of the redundant mucosa, the mucosal edges were sutured to the meatus using fine absorbable sutures (4-0 polyglycolic acid, Safil QuickTM, B. Braun Melsungen AG) at the 3, 6, 9 and 12 o'clock positions. A 12 Fr Foley catheter was left in place for $24 \mathrm{hrs}$ and the patient was advised to refrain from spicy food and alcohol consumption for the following 10 days.

\subsubsection{Fulguration of genital warts}

Genital warts (HPV lesions, condylomata acuminata) were treated with fulguration in 48 young patients ( 42 male and 6 female, mean age \pm SD: 23.9 yrs \pm 3.3 ). In the males the location of the warts varied: in most cases they involved the penile shaft skin and the preputial ridge, the inner layer of the prepuce, the frenular region and in 3 cases the glans penis. In the females the lesions were located at the vulvar region and in one case at the perineum. In cases of mucosal lesions topical anesthesia was achieved using a small quantity of EMLA cream (approximately 1-3 gr, depending on the extent of the lesions) and an occlusive dressing to keep the cream from being dislodged (Fig. 3). No dressing was used when the lesions were near hairy skin. In the cases of penile skin lesions and the perineal warts the dressing was left in place for 1 hour. The procedure was completed by fulguration of all visible lesions using the Birtcher Hyfercator at low power settings (approx. 5 Watts).

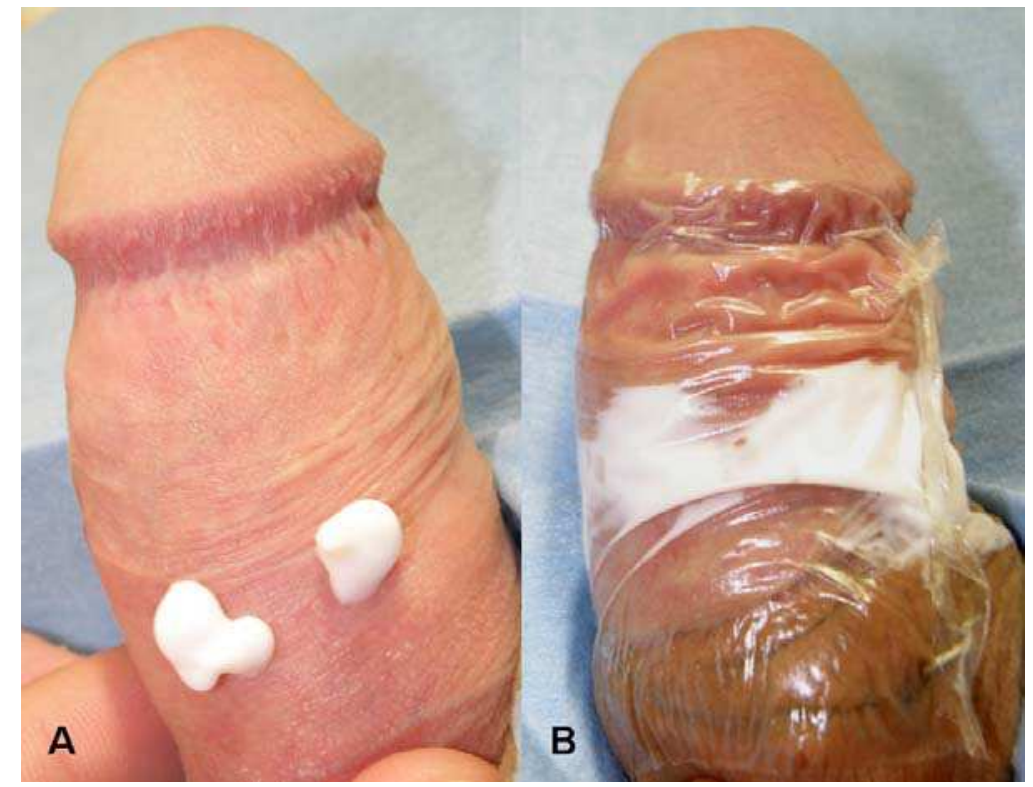

Fig. 3. Topical application of EMLA on penile HPV lesions (A). Placement of occlusive dressing (B). 


\subsubsection{Circumcision}

A total of 68 male patients (mean age \pm SD: $47.8 \mathrm{yrs} \pm 24.7$ ) were subjected to circumcision due to phimosis. Topical anesthesia was initially achieved by spreading EMLA cream (approx. 2-5 gr) in the preputial sac with the aid of a $5 \mathrm{ml}$ syringe. A plain cling film dressing was used for preventing the cream to spread out and the level of analgesia was evaluated first at 20 minutes and again at 30 minutes by pinching the prepuce with a toothed forceps. If the patient still reported pain or discomfort, further anesthesia was achieved initially by local infiltration with lignocaine (Xylocaine 2\%, AstraZeneca Inc) at the ventral area (the frenular area). If this measure was inadequate, dorsal penile block with approximately $20 \mathrm{ml} \mathrm{1 \%} \mathrm{Xylocaine} \mathrm{was} \mathrm{performed.} \mathrm{After} \mathrm{complete} \mathrm{anesthesia} \mathrm{was}$ achieved, the foreskin was elevated, clamped with a straight forceps and cut until the glans was freed. Any redundant skin and inner prepuce were trimmed. Haemostasis was meticulously performed with diathermy and finally the edges of the skin and inner prepuce were sutured using absorbable sutures (3-0 polyglycolic acid, Safil QuickTM, B. Braun Melsungen AG). After completion of the procedure the traumatic surface was smeared with povidone iodine cream and a circular gauge dressing was applied leaving the urethra meatus free. The patient was advised on daily changes of the dressing and extra care of personal hygiene. No antibiotics or analgesics were prescribed.

\section{Results}

\subsection{Effectiveness of topical anesthesia}

A total of 321 patients were subjected to minor surgery of the external genitalia under topical anesthesia with EMLA cream. With the exception of circumcision patients, only one case in the short frenulum group and four cases in the genital warts group required further infiltration with local anesthetic. The overall satisfaction rate was excellent, as all patients were enthusiastic with the absence of needle pricks. All patients to whom anesthesia was sufficiently obtained with topical cream application stated that they would opt again for this type of local anesthesia for a similar procedure in the future. Detailed results for each type of procedure are presented below.

\subsubsection{Separation of preputial adhesions}

In all patients the use of EMLA cream obtained a sufficient level of anesthesia, allowing for completion of the procedure without need for further local anesthetic. However, anxiety of younger children did not allow for complete estimation of the level of local anesthesia, as we did not use formal monitoring of physiological parameters such as heart rate, respiratory rate etc. Nevertheless, we did not observe major symptoms of distress (excessive crying, protective movements) even in young boys ( $<20$ months).

\subsubsection{Short frenulum plasty}

The level of local anesthesia obtained by EMLA cream in 20 minutes was sufficient in all cases except one. The latter was a patient with a notably thick frenulum who required further local anesthesia with lignocaine infiltration $(\sim 0.5 \mathrm{ml}$ Xylocaine $2 \%)$ down the course of the procedure, while the deeper plate of the mucosa of the frenular bed was fulgurated for haemostasis. With the "pull and burn" method no sutures were generally used; in only 
three patients $(2 \%)$ with a thick and wide frenulum, fine absorbable sutures (5-0 polyglycolic acid, Safil Quick ${ }^{\mathrm{TM}}$, B. Braun Melsungen AG) were used to approximate the lateral edges of the tear in order to achieve faster wound healing and better cosmetic result.

\subsubsection{Meatotomy}

The use of EMLA cream was in all cases sufficient to obtain a satisfactory level of local anesthesia until completion of the procedures. Even when sutures were used to anchor the urethral mucosa at the meatal lips, no pain was reported by the patients.

\subsubsection{Fulguration of urethral warts}

Topical EMLA application achieved a complete level of local anesthesia in all patients. Even when meatotomy was performed in order to gain access to warts rooted deeply in the fossa navicularis, no further anesthesia was necessary.

\subsubsection{Urethral prolapse correction}

The intraurethral application of EMLA cream was in all cases sufficient to obtain a satisfactory level of local anesthesia until completion of the procedures. Only one woman reported minor discomfort while the urethral mucosa was sutured to the meatus; however this was well tolerated and no further infiltrative anesthesia was required.

\subsubsection{Fulguration of genital warts}

Generally, warts on mucosal surface (penile, vulvar) were removed under topical cream anesthesia. In 4 out of 9 cases $(45 \%)$ of warts located on penile and perianal skin further infiltration with $2 \%$ lignocaine was required. However in three of these cases which were men with condylomata located at the penile shaft skin near the pubis, the occlusive dressing had been dislocated during the waiting time of 1 hour, as pubic hair did not allow for airtight adhesion of the Tegaderm ${ }^{\circledR}$ dressing. In the rest of 44 patients $(91.67 \%)$ no further local anesthetic was required and fulguration was completed uneventfully.

\subsubsection{Circumcision}

In only $14 / 68$ cases $(20.5 \%)$ with phimosis the use of topical cream alone was sufficient for anesthesia until completion of the procedure. Although not properly classified, our impression is that these patients featured a limited area of foreskin, which was thin and elastic. In the rest of the 54 patients $(79.5 \%)$ further local infiltration with $2 \%$ lignocaine was required in 21 cases (38.9\%) and dorsal penile nerve block with $1 \%$ lignocaine was required in 33 cases $(61.1 \%)$. The use of EMLA cream in most of these cases would alleviate to some extent the painful stimuli (e.g. clamping with forceps); however this was not adequate for completion of the procedure and the patients required further analgesia.

\subsection{Complications and side effects}

A sensation of "mint freshness" was reported by most patients when the cream was applied on mucosal surface. Local erythema was present in almost all cases with mucosal 
application; however this did not affect the course of the procedures. No significant skin reaction or allergic reaction occurred in any patient. No systematic reactions, cyanosis or methemoglobinaemia were recorded, even in the group of young boys undergoing separation of preputial adhesions.

\section{Discussion}

The development of topical anesthetics in the latter half of the $19^{\text {th }}$ century was a revolution in local anesthesia. Today pain can be alleviated for a wide variety of minor procedures on intact or even lacerated skin and mucosa. This evolution in topical cream anesthetics has offered surgeons, plastic surgeons and even family medicine physicians a useful tool in treating several conditions safely at an office setting. In the genitourinary surgery setting, these procedures include (but are not limited to): circumcision, preputial plasty and frenulum plasty, meatotomy and meatal dilatation, removal of genital warts by curettage, fulguration, laser surgery or cryotherapy, biopsies of genital skin and mucosa, separation of preputial adhesions in boys and hymenal or labial adhesions in girls. In the past, most types of these procedures would require infiltration with a local anesthetic such as lignocaine and at least some form of sedation. The use of topical acting anesthetics is patient-friendly as they eliminate needle fear and anxiety and injection pain at the beginning of the procedure. Moreover the minor skin reactions observed (local erythema or minor oedema) are an advantage over infiltrative anesthetics which often produce bleeding, heamatomas or oedema that may distort the surgical site, a fact that may be important in delicate procedures with a desired good aesthetic result. One should also bear in mind the always present risk of accidental systemic intravascular injection of injectables, especially in procedures where higher doses of local anesthetic are needed. In this series we have used EMLA cream, a eutectic mixture of local anesthetics, $2.5 \%$ lidocaine and $2.5 \%$ prilocaine, to provide local anesthesia for a variety of surgical procedures at the external genitalia of children, men and women at an office setting.

\subsection{Topical anesthetics}

EMLA has not been the first topical anesthetic to be used in the office setting. After the initial enthusiasm for cocaine as a topical anesthetic, a series of mixtures have been developed in an effort to produce an effective and safe topical compound. Tetracaine, adrenaline and cocaine (TAC), a compound of $0.5 \%$ tetracaine, $0.05 \%$ epinephrine and $11.8 \%$ cocaine was the first mixture of topical anesthetics to be effective for skin lacerations (Kundu, 2002). A small amount of this solution $(2-5 \mathrm{ml})$ is applied directly to the wound with a cotton tip and maintained under pressure for 20-40 minutes. However concerns about toxicity, expence and legal issues regarding cocaine have led the medical community to abandon its use (Kundu, 2002).

An alternative to TAC (with cocaine substituted by lignocaine) was LET, a compound of $0.5 \%$ tetracaine, $0.1 \%$ epinephrine and $4 \%$ lignocaine (Xylocaine). This mixture has to be formulated as a liquid or gel. No severe side-effects have been reported but care should be taken when used in end-arterioral areas of the body due to the effect of epinephrine. Moreover, mucosal application should be avoided. Other newer compounds developed include LMX-4 and LMX-5 (which contain liposomal lignocaine $4 \%$ or $5 \%$ respectively) and 
BLT (a triple anesthetic gel consisting of $20 \%$ benzocaine, $6 \%$ lignocaine and $4 \%$ tetracaine). Iontophoresis is another method that can transfer medications into the skin through the use of constant, low-voltage direct current. In this setting, sponges soaked in lignocaine are applied on intact skin and the current is applied, producing a good anesthetic effect that lasts for 42 to 110 minutes (Kaweski, 2008). However there are limits to this method too. Skin burns may occur if the current is set too high, special equipment and training are necessary and the area to be anaesthetized can only be small and flat. Moreover, it has not been used on mucosal surfaces.

EMLA cream is an oil-in-water emulsion of $2.5 \%$ lignocaine and $2.5 \%$ prilocaine. The eutectic mixture contains a thickener, an emulsifier and distilled water adjusted to a $\mathrm{pH}$ level of 9.4 (Kundu, 2002). This formulation enhances absorption through intact skin. For our group of patients EMLA seemed the best choice, due to its commercial availability, low cost and easiness in application. Moreover EMLA has been successfully used on genital mucosa, despite initial concerns of prilocaine absorption. It should be noted however that other topical anesthetics may give comparable results as they all share common action mechanisms.

\subsection{Mechanism of action}

For a topical anesthetic to act it must firstly penetrate the outer barriers of the body, eg the epidermis or the mucosal surface in order to reach the deeper dermal layers of the skin, concentrate in the vicinity of pain receptors and free nerve endings and eventually penetrate the individual axons in the nerve. Local anesthetics generally feature a lipid-soluble hydrophobic aromatic group (aromatic ring), an intermediate ester and a charged, hydrophilic group (terminal amine). Greater lipid solubility enhances diffusion through nerve sheaths as well as neural membranes of individual axons. Both lignocaine and prilocaine contained in EMLA cream are amide type anesthetics, which accounts for their stability, heat resistance and low allergenic potential (Edgcombe, n.d.). These local anesthetics act through interruption of the neural conduction through inhibition of the influx of sodium ions (Becker, 2006).

Normal neural conduction is based on the ability of neural tissue to become excitable in an electrical sense and further conduct the initial impulse. This is mediated through fluctuations of the ionic gradients that normally exist across the axonal membrane. At the resting phase, a potential of about $70 \mathrm{mV}$ (resting potential) is generated by the constant activity of a sodium-potassium pump which continuously pumps out sodium to the outside of the membrane while potassium is being pumped in. The result is marked concentration difference (concentration gradient) for these two cations across the axonal membrane (Neilson, n.d.). Depolarisation of the membrane is triggered by sudden opening of sodium channels, resulting in a massive influx of $\mathrm{Na}$. This in turn produces a rise in membrane potential to around $40 \mathrm{mV}$. Repolarisation occurs through closure of sodium channels in the presence of continuous efflux of potassium. As the intracellular potential falls back to its resting levels, the potassium channels close in turn. This depolarization wave is propagated and the impulses are transmitted across the axon.

Local anesthetics disrupt ion channel function within the neuronal membrane. This is occurring by specific binding of the anesthetic molecule to the sodium channels. The 
channels are held in an inactive state, preventing further depolarization of the membrane. The action of the molecule in its ionized form is exerted from within the cell i.e. the anesthetic has to cross the neuronal membrane in order to block the sodium channels. The effect of the anesthetic is not limited to pain reduction; the loss of nerve function also includes loss of temperature, touch, proprioception and eventually muscle tone (Edgcombe, n.d)

The time for onset of local anesthesia is regulated by the amount of unionized drug present at physiological $\mathrm{pH}$ (7.4). As all local anesthetics are weak bases, they exist in both ionized and unionized form. The dissociation constant $(\mathrm{pKa})$ of a local anesthetic determines the amount of an administered dose that exists in an ionized form at any given $\mathrm{pH}$. The lower the $\mathrm{pKa}$, the greater the proportion in the tertiary, diffusible state, a fact that in turn hastens onset of action. Lignocaine for example has a pKa of 7.9 while bupivacaine has a pKa of 8.1, hence lignocaine is approximately $25 \%$ (versus $15 \%$ for bupivacaine) unionized at $\mathrm{pH} 7.4$, a fact that explains lignocaine's more rapid onset of action. Prilocaine has also a pKa of 7.9, hence the formulation of EMLA has two equally fast acting local anesthetics.

\subsection{Application}

The method of application of EMLA cream is straightforward and easy. According to the product datasheet, a thick layer of cream is applied on intact skin and covered with an occlusive dressing, either a self-adhesive e.g. Tegaderm ${ }^{\circledR}$ or plain cling film. The purpose is to create an air-tight environment that facilitates absorption of the cream ingredients. After minimum one hour $(1 \mathrm{hr})$ the dressing is removed and any excess cream has to be cleaned thoroughly prior to the procedure. For skin applications the suggested dose is $1.5 \mathrm{gr} / \mathrm{cm}^{2}$ and maximum application time is 5 hours. For mucosal applications, the suggested dose is even higher, approximately $2 \mathrm{gr}$ per lesion, with a maximum of $10 \mathrm{gr}$. The suggested waiting time is 5-10 minutes. An occlusive dressing is not necessary (EMLA product monograph).

In our cases with skin lesions such as penile skin warts and circumcision we used the recommended dose for 1 hour in all cases, along with an occlusive dressing. Other investigators have used even higher doses on skin applications (Laffon, 1998; Buckley \& Benfield, 1993). When treating lesions on mucosal surfaces (penile or vulvar) we used the smallest quantity necessary and not the recommended dose. An occlusive dressing was used on mucosal surfaces as well (either Tegaderm ${ }^{\circledR}$ or plain cling film) although not suggested. The reason for the dressing was not to create an air-tight barrier but rather to prevent the cream from being dislodged while the patient relaxed in the waiting room. In cases of vulvar lesions where no dressing was applicable the patients had to wait in the office surgery, usually in a modified lithotomy position. The time allowed for the cream to act on mucosal surfaces was generally 20 minutes. The recommended time is $5-10$ minutes; however from our previous experience this amount of time is not always sufficient for achieving a satisfactory level of local anesthesia, especially for procedures such as frenulum plasty or large condylomata in which deeper levels of the preputial mucosa had to be fulgurated. Other studies have suggested that the quality of analgesia actually decreases with application times longer than 10 to 15 minutes (Zilbert, 2002). This decreased efficacy associated with longer periods of application time is attributed to lidocaine-induced 
vasodilatation and subsequent increased systemic absorption (Kundu, 2002). However this negative time effect has not been verified in our series.

When the EMLA cream was delivered into the urethral meatus (e.g. in urethral warts, meatotomy and urethral prolapse) we used a small syringe to administer the desired dose. This was much easier than using small probes or cannulation catheters we had used in the past. One has to be careful to remove all air bubbles from the syringe before squeezing the cream. A time lap of 20 minutes was in all cases sufficient for achieving an adequate level of anesthesia.

The use of EMLA in the pediatric population warrants special precautions. The suggested dose for infants 3 up to 12 months is up to 2 gr, for approximately 1 hour. For children 1-6 years and $>10 \mathrm{~kg}$ the dose increases up to $10 \mathrm{gr}$. However these refer to skin applications (EMLA product monograph). No standardized doses for mucosal applications are available. However several studies have used EMLA cream for topical anesthesia in the preputial sac, for procedures such as circumcision, meatotomy and release of preputial adhesions (Smith, 2004; Ben-Meir, 2011; Butler-O'Hara, 1998). The doses used varied from 0.5 to $2 \mathrm{gr}$ and the application time extended from 30 mins to 1 hour. In our cases of preputial adhesions we used a cotton tip to deliver the cream inside the preputial sac and tried to use the minimum amount possible $(0.5-1 \mathrm{gr})$. In most cases the anesthetic effect was already demonstrable at 20 mins.

\subsection{Efficacy}

In our series the EMLA cream provided excellent topical anesthesia when mucosal surfaces were treated. In the majority of adult cases where mucosal lesions were involved, local anesthesia was thorough and allowed for completion of the procedure without the need for further infiltrative anesthetic. Only one patient with an extra thick frenulum required further lignocaine injection during the procedure. Our results verify the excellent anesthetic effect of EMLA on the genital mucosa. Similar results have been demonstrated with EMLA use in procedures such as frenuloplasty or meatotomy (Laffon, 1998; Buckley \& Benfield, 1993; Ben-Meir, 2011). However, the above mentioned results are not directly comparable, as the above mentioned investigators also used other forms of analgesia or sedation (e.g. nitrous oxide).

When EMLA was applied on skin lesions, the results were not so impressive. In 4 cases with genital warts the degree of anesthesia was insufficient and further local anesthetic ( $2 \%$ Xylocaine) had to be injected on site. Of course a "relieving" explanation in three of these cases would be the dislodgment of the cream from the penile skin; however even in these cases enough cream was left in place for a positive result to occur. Failures were most prominent in the group of phimosis patients undergoing circumcision. In only $20.5 \%$ of these patients topical anesthesia was adequate for completion of the procedure. In the majority of the circumcision cases $(79.5 \%)$ additional local infiltration with $2 \%$ Xylocaine or dorsal penile nerve block with $1 \%$ Xylocaine was necessary. Similar results have been reported by other authors in both adult and pediatric populations undergoing circumcision (Laffon, 1998; Butler-O'Hara, 1998) .It appears that the complex innervation of the penile skin and prepuce does not allow for the use of a topical cream as a sole anesthetic agent. In the case of a proper circumcision, three parts of the penis have to be anesthetized: the penile 
shaft skin, the inner layer of the prepuce and especially the ridged band and the mostly sensitive frenular area. This may explain why application of EMLA on the inner part of the preputial sac is firstly incomplete (due to phimosis) and secondly inadequate to provide anesthesia to the penile shaft skin. Even combined application (i.e. administration to both the preputial sac and the shaft skin with occlusive dressing) has not been proved adequate (personal unpublished data). It is of interest that only in cases with a thin, soft prepuce (although this can not be properly measured) the EMLA cream provided sufficient analgesia. It is possible that penetration of the anesthetics was easier through a thinner mucosal epithelium and lamina propria of the prepuce. Nevertheless it should be noted that although EMLA was insufficient for initiation or completion of the procedure it did however partly alleviate the pain from further local infiltration. This finding suggests that EMLA may be used as an agent for pain reduction associated with local infiltration or dorsal penile nerve block during circumcision.

In our pediatric population the effect of EMLA on pain reduction during release of preputial adhesions is deemed effective. Although we did not use proper tools such as Visual Analog Scale (VAS), the Wong-Baker Faces scale or Neonatal Infant Pain Scale (NIPS), behavioral responses (facial activity, time spent crying, protective movement) were recorded during the procedures. However it is rather difficult to correctly estimate the effect of EMLA in this setting as the anxiety of certain young patients (or the anxiety of the parents!) may obscure the effect of the local anesthetic. In our series every care was taken to reassure the patients that no pain would be present and that they just had to stay relaxed and tranquil. The effect of the parents' presence was equivocal, depending on the character of the child. Keeping these variables in mind, we considered the topical anesthetic effective when no major signs of distress (excessive crying, protective movements, quick breathing) were present during the procedure and the patient himself tolerated the procedure until completion.

\subsection{Adverse effects}

No major adverse effects were present in any case in this series. In the adult population only topical signs of a transient erythema were present in almost all cases of mucosal application. This biphasic skin reaction of blanching and erythema is thought to occur due to initial peripheral vasoconstriction followed by vasodilatation (Kaweski, 2008). In any case this is a negligible side effect that does not affect the surgical site. Most of our patients reported that the erythema disappeared within 1-2 hours. A mild sensation of burning and itching has also been reported in several studies (Zilbert, 2002). In our series most patients only reported a "freshmint" sensation at the site of application. Rarely contact dermatitis may develop secondary to prilocaine (Kaweski, 2008). Another rare but potentially lethal complication of local anesthetics is methemoglobinemia.

Methemoglobin (metHb) is produced when within the hemoglobin molecule ferrous iron is oxidized to ferric iron. This effect impairs oxygen transport to tissues (Boran, 2008). $\mathrm{MetHb}$ is normally maintained at concentrations below $2 \%$ of total hemoglobin, due to the activity of NADH dehydrogenase which reduces metHb to hemoglobin. Neonates are most prone to develop methemoglobinemia because the cytochrome b5 reductase level in infants is only $50 \%$ of adult values and methemoglobin can accumulate. Several drugs 
may induce methemoglobinemia, such as sulfonamides, nitrofurantoin, nitrates, phenytoin, phenacetin, phenobarbital and others. As far as EMLA is concerned, a metabolite of prilocaine called o-toluidine is known to induce methemoglobinemia (EMLA product monograph).

The initial presentation of an infant with mild methemoglobinemia is characterized by tissue cyanosis, a bluish-grey discoloration of the skin, especially around lips and nail beds, palor or marbleization. It is characteristic that this cyanotic picture is not reversed by administration of $100 \%$ oxygen. Severe methemoglobinemia (concentrations above 25\%) presents with seizures, cardiovascular collapse and coma. Severe methemoglobinemia represents an emergency; first-line antidote is methylene blue, a thiazide dye that accelerates the enzymatic reduction of metHb by NADHP reductase and also acts as scavenger for free radicals (Boran, 2008). However when methylene blue is not readily available, intravenous high doses of ascorbic acid may be used as an alternative.

\subsection{Safety concerns}

Topical anesthetic creams are generally safe and effective when used appropriately. In the past deaths have occurred when anesthetic creams compounded in formulas with nonstandard doses were used by inexperienced personnel or even self-applied by patients (Kaweski, 2008). EMLA cream is a standard dose compound, with many years of clinical use. The recommended doses should be respected and the application time not exceeded significantly. In our series we used the smallest amount possible, especially on mucosal application. The recorded effectiveness of EMLA cream in these cases, even with doses smaller than suggested, demonstrates that the anesthetic effect is not necessarily associated with a "thick layer" of cream, as originally described in the product's datasheet (EMLA product monograph). Every effort should be made to limit the skin or mucosal area covered to the smaller extent necessary. EMLA cream is not intended for use on large areas. Special precautions should be used in specific cases. A history of congenital or idiopathic methemoglobinemia is an absolute contraindication; patients with glucose-6-phosphate dehydrogenase (G-6-P-D) deficiency and those who require treatment with methemoglobin - inducing drugs are more susceptible to acquired methemoglobinemia.

The use of EMLA in the pediatric population should follow the general rules on dosage and application time with a special focus on the increased vulnerability of infants to methemoglobinemia. Topical use of EMLA should be avoided in infants less than 3 months and other sedation or anesthetic alternatives should be preferred for this age group. Moreover, when EMLA cream is used for topical anesthesia, children should be closely monitored during and after the procedure for early signs of complications such as methemoglobinemia. In our series no such an event occurred. Nevertheless the children with preputial adhesions were kept and monitored in the waiting room for at least one hour after completion of the procedure and the parents and caretakers were thoroughly advised on which signs they should become alert during the following hours.

\section{Conclusions}

The use of topical anesthetic creams has gained popularity in the recent years over injectable local anesthetics due to the advantage of needle pain and anxiety elimination. In our series 
of patients the application of EMLA cream as a topical anesthetic proved to be a useful, efficient and safe tool for minor surgical procedures of the external genitalia in children, men and women at the office setting. Side effects can be kept to a minimum when the amount used is limited (especially at mucosal application) and the time allowed for action is carefully tailored to the site of application, patient's age and type of procedure.

\section{References}

Becker D, Reed K. Essentials of local anesthetic Pharmacology. Anesth Prog Vol. 53, (2006), pp. 98-108.

Ben-Meir D, Livne PM, Feigin E, Djerassi R. \& Efrat R. Meatotomy using local anesthesia and sedation or general anesthesia with or without penile block in children: a prospective randomized study. J Urol Vol 185, No.2, (Feb 2010), pp. 654-657.

Boran P., Tokuc G., \& Yegin Z. methemoglobinemia due to application of prilocaine during circumcision and the effect of ascorbic acid. Journal of pediatric Urology. Vol.1 No4 (2008), pp.475-476.

Buckley M., Benfield P. Eutectic Lidocaine/Prilocaine cream: A review of the topical anaesthetic/analgesic efficacy of a Eutectic Mixture of Local Anaesthetics (EMLA). Drugs, Vol.46, No.1 (July 1993), pp.126-151.

Butler-O'Hara M, LeMoine C. \& Guillet R. Analgesia for neonatal circumcision: arandomized controlled trial of EMLA cream versus dorsal penile nerve block. Pediatrics, Vol. 101, No 4, (1998), e5.

Cold CJ, Taylor JR. The prepuce. British Journal of Urology, Vol 83, Suppl. No 1, (1999), pp. 34-44.

Edgcombe H. Local anaesthetic pharmacology. Available from: http://www.frca.co.uk/ printfriendly.aspx?articleid=100505

EMLA product monograph. Date of revision: May 5, 2010. Available from: http://www.astrazeneca.ca.

Gordetsky J, Bendana E, OBrien J, \& Rabinowitz R. (Almost) painless surgery: a historical review of the evolution of intraurethral anesthesia in Urology. Urology, Vol.77, No 1, (2011), pp.12-16.

Gyftopoulos K. Male dyspareunia due to short frenulum: the suture-free, "pull and burn" method. Journal of Sexual Medicine Vol.6, No9 (September 2009), pp. 2611-2614.

Kaweski S. Topical Anesthetic creams. Plastic E Reconstructive Surgery Journal. Vol.121, No.6, (June 2008), pp. 2161-6165.

Kundu S, Achar S. Principles of office anesthesia: PartII. Topical anesthesia. American Family Physician. Vol.66, No1, (July 2002), pp. 99-102.

Laffon M, Gouchet A, Quenum M, Haillot O, Mercier C. \& Huguet M. Eutectic mixture of local anesthetics in adult urology patients: an observational trial. Regional Anesthesia Pain Medicine. Vol. 23, No.5, (Sep-Oct 1998), pp. 502-5.

Neilson A. Mechanism of action of local anaesthetics. Available from: http://www.csaol.cn/img/hypertextbook/a/c53.htm

Smith DP, Gjellum M. The efficacy od LMX versus EMLA for pain relief in boys undergoing office meatotomy. J Urol Vol 172, No 4, (Oct 2004), pp. 1760-1761. 
Zilbert A. Topical anesthesia for minor gynaecological procedures: A review. Obstetrical and Gynaecological Survey. Vol57, No.3, (2002), pp. 171-178. 


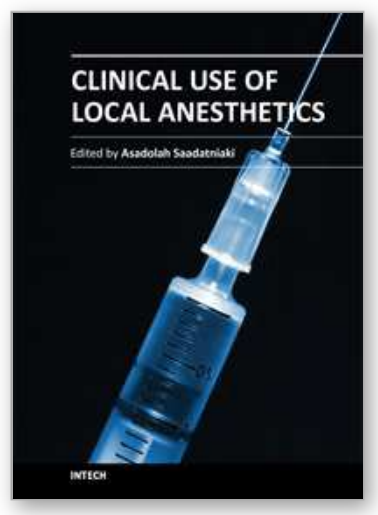

\author{
Clinical Use of Local Anesthetics \\ Edited by Dr. Asadolah Saadatniaki
}

ISBN 978-953-51-0430-8

Hard cover, 102 pages

Publisher InTech

Published online 23, March, 2012

Published in print edition March, 2012

Local anesthetics are being increasingly applied in different surgeries. Lower side effects of neuroaxial anesthesia, regional anesthesia, and field block, in comparison to general anesthesia (volatile and intravenous agents), are the main reasons why physicians prefer to conduct surgeries under local anesthesia, especially in outpatient and day care surgeries. It is important to emphasize the presence of an anesthesiologist, and vigilant monitoring of the homodynamic parameters, in decreasing a patient's anxiety, exerting other modalities for analgesia and increasing the safety margin in many procedures.

\title{
How to reference
}

In order to correctly reference this scholarly work, feel free to copy and paste the following:

Kostis Gyftopoulos (2012). The Use of Topical Cream Anesthetics in Office Procedures of the External Genitalia, Clinical Use of Local Anesthetics, Dr. Asadolah Saadatniaki (Ed.), ISBN: 978-953-51-0430-8, InTech, Available from: http://www.intechopen.com/books/clinical-use-of-local-anesthetics/the-use-of-topical-creamanaesthetics-in-office-procedures-of-the-external-genitalia-

\section{INTECH}

open science | open minds

\section{InTech Europe}

University Campus STeP Ri

Slavka Krautzeka 83/A

51000 Rijeka, Croatia

Phone: +385 (51) 770447

Fax: +385 (51) 686166

www.intechopen.com

\section{InTech China}

Unit 405, Office Block, Hotel Equatorial Shanghai

No.65, Yan An Road (West), Shanghai, 200040, China

中国上海市延安西路65号上海国际贵都大饭店办公楼 405 单元

Phone: +86-21-62489820

Fax: +86-21-62489821 
(C) 2012 The Author(s). Licensee IntechOpen. This is an open access article distributed under the terms of the Creative Commons Attribution 3.0 License, which permits unrestricted use, distribution, and reproduction in any medium, provided the original work is properly cited. 